\title{
Bound state representation of scattering solutions*
}

\author{
W. H. Bassichis, J. F. Reading, and R. R. Scheerbaum \\ Department of Physics and Cyclotron Institute, Texas A\&M University, College Station, Texas 77843
}

(Received 24 July 1974)

\begin{abstract}
Approximate positive energy continuum eigenstates of a one-body Hamiltonian, already projected onto a finite dimensional harmonic oscillator basis, are constructed from the set of discrete-energy states obtained by diagonalizing the Hamiltonian. The method should be especially useful for calculating the virtual, continuum, intermediate states in manyparticle calculations. The results for the test cases considered, the square well and the nonlocal Hartree-Fock potentials, are quite accurate both in the wave functions and the phase shifts.
\end{abstract}

\section{INTRODUCTION}

One of the more fruitful methods used in trying to solve the nuclear many-body problem has as its starting point the solution of the Hartree-Fock equations in a truncated, harmonic oscillator basis. ${ }^{1}$ If the nucleus under consideration has $A$ particles and the basis consists of $N$ states, one generates, by this method, $N-A$ "unoccupied" eigenfunctions as well as the filled states. The HartreeFock (HF) representation thus obtained is in many cases the most convenient one for subsequent calculations. For example, in applying the random phase approximation method to describe excited states, the use of the HF states circumvents the usual difficulty with the elimination of spurious states. $^{2}$ These states are, in this case, not mixed with the real excited states and have zero excitation energy. Also, when carrying out a perturbation expansion the $\mathrm{HF}$ representation leads to a great simplification; namely, that the one particleone hole states are not connected by the Hamiltonian to the unperturbed ground state.

Because all such nuclear calculations ultimately involve the calculation of two-body matrix elements, the harmonic oscillator basis is particularly convenient since the decomposition of two states into relative and center -of-mass states, i.e., the Talmi or Brody-Moshinsky transformation, ${ }^{3}$ consists of a limited number of terms. Sophisticated and efficient computer programs enable one to calculate extremely large numbers of two-body, harmonic oscillator matrix elements and render large HF calculations, even of superheavy nuclei, and subsequent perturbation calculations quite feasible.

Thus, since the results of large HF calculations are available and because of the consequent simplicity of calculation, it was desirable to develop a procedure for expressing scattering states in terms of $\mathrm{HF}$ states given in a harmonic oscillator basis. The quite complicated expressions occur- ring in perturbative scattering schemes, where complete sets of scattering wave functions are introduced as intermediate states, would then be calculable using essentially the same method employed for the bound states. Such a procedure is described in the next section. Of course, for the procedure to be ultimately useful it is necessary to demonstrate that only a few harmonic oscillator states are needed to accurately describe the scattering wave function inside the potential region. In Sec. III the method is tested for two exactly soluble models and it is shown that this is the case.

It should be emphasized that the method is similar in spirit to the $R$-matrix approach ${ }^{4}$ and the method of Heller and Yamani. ${ }^{5}$ It differs from the $R$-matrix procedures in that it gives the scattering solution over the range of interest and not just the phase shifts. Furthermore, and of crucial importance, the required calculations are straightforward and not time consuming, even for nonlocal potentials.

Finally, it should be noted that the method is applicable whenever one can diagonalize a one-body Hamiltonian in a harmonic oscillator basis. The method is described in terms of HF results because of the subsequent application, but may be used with other single-particle model results, such as Nilsson calculations.

\section{METHOD}

When solving the HF equations in a truncated, harmonic oscillator basis, one diagonalizes the matrix

$$
h_{i j}=t_{i j}+u_{i j} \equiv\langle i|t+u| j\rangle,
$$

where $u$ is the HF potential defined by

$$
\langle i|u| j\rangle=\sum_{\lambda}\left\langle i \lambda\left|V_{A}\right| j \lambda\right\rangle \text {. }
$$

Here $|i\rangle$ and $|j\rangle$ are harmonic oscillator states and the $|\lambda\rangle$ are the occupied HF orbitals which are 
expanded in terms of $|i\rangle$ :

$$
|\lambda\rangle=\sum_{i} C_{i}^{\lambda}|i\rangle \text {. }
$$

$V_{A}$ is the antisymmetrized two-body interaction. At present only spherical nuclei shall be considered so that $|\lambda\rangle$ is an eigenstate of orbital and total angular momentum and isospin. The HF equation is then

$$
\sum_{n^{\prime}} h_{n n^{\prime}} C_{n^{\prime}}^{\lambda}=\epsilon_{\lambda} C_{n}^{\lambda}
$$

where $n$ and $n^{\prime}$ are principal quantum numbers and one has a separate matrix for each $l$ and $\vec{j}$.

In order to emphasize that a truncated basis is being used, projection operators may be defined by

$$
\begin{aligned}
& P=\sum_{i=1}^{N}|i\rangle\langle i|, \\
& Q=\sum_{i=N^{+}}^{\infty}|i\rangle\langle i|, \\
& P+Q=1,
\end{aligned}
$$

and the HF equation takes the form

$$
P h P|\mu\rangle=\epsilon_{\mu}|\mu\rangle \text {, }
$$

where the $N$ HF states $\mu$ are, of course, in the $P$ space.

It is apparent from Eq. (6) that this diagonalization method will never yield, directly, correct scattering (positive $\epsilon$ ) solutions. Although the potential term may be well represented by $P u P$, the kinetic energy operator has matrix elements which increase with principal quantum number $n$. It is this part of $h$ which is nonzero at infinity and will give rise to the continuum. Thus the correct equation for the scattering states must include the entire space

$$
(P+Q) h(P+Q)\left|\psi_{k}\right\rangle=\epsilon_{k}\left|\psi_{k}\right\rangle .
$$

The boundary condition is such that in the coordinate representation the radial part of $\psi_{k}(r)$ is given asymptotically by

$$
R_{k l}(r) \sim j_{l}(k r)-\tan \delta_{l} n_{l}(k r)
$$

for any particular $l$ value.

One may now, in the standard way, combine Eqs. (6) and (7) by multiplying the former by $\left\langle\psi_{k}\right|$ and the latter by $\langle\mu|$. Subtraction then yields

$$
\left\langle\mu|P h Q| \psi_{k}\right\rangle=\left(\epsilon_{k}-\epsilon_{\mu}\right)\left\langle\mu \mid \psi_{k}\right\rangle,
$$

if $u$, which may be nonlocal or complex, is at least symmetric. The first real assumption in the method is now introduced. It will be assumed that the basis is sufficiently large so that $u$ may be well represented in it, i.e.,

$$
\begin{aligned}
u\left(\overrightarrow{\mathrm{r}}, \overrightarrow{\mathrm{r}}^{\prime}\right) & =\sum_{i, j=1}^{\infty}\langle\overrightarrow{\mathrm{r}} \mid i\rangle\langle i|u| j\rangle\left\langle j \mid \overrightarrow{\mathrm{r}}^{\prime}\right\rangle \\
& \approx \sum_{i, j=1}^{N}\langle\overrightarrow{\mathrm{r}} \mid i\rangle\langle i|u| j\rangle\left\langle j \mid \overrightarrow{\mathrm{r}}^{\prime}\right\rangle .
\end{aligned}
$$

The validity of this assumption obviously cannot be established mathematically as it is trivial to construct nonlocal potentials for which $P u Q$ is not negligibly small no matter how large a basis is used. However, for the physical systems of inter est it is reasonable that, due to the smooth and slowly varying nature of $u$, Eq. (10) will be well satisfied. Alternatively, one may adopt the approach that the matrix elements of $u$ which are available from $\mathrm{HF}$-type calculations in oscillator space will always be in a truncated basis and there is little choice but to assume that those out of the space are zero. ${ }^{6}$

One now uses the well-known property of the matrix elements of the kinetic energy between harmonic oscillator states, namely that all quantum numbers except $n$ must be the same and the principal quantum numbers can differ by at most unity in order for the matrix element to be nonzero. Then assuming, for notational simplicity, that there are $\eta$ basis states for each $l$ and $\vec{j}$ in the $P$ space, one obtains for the left side of Eq. (9)

$$
\begin{aligned}
\left\langle\mu|\operatorname{PtQ}| \psi_{k}\right\rangle & =\sum_{i=\eta+1}^{\infty}\langle\mu|t| i\rangle\left\langle i \mid \psi_{k}\right\rangle \\
& =C_{\eta}^{\mu}\langle\eta|t| \eta+1\rangle\left\langle\eta+1 \mid \psi_{k}\right\rangle
\end{aligned}
$$

so that

$$
\left\langle\mu \mid \psi_{k}\right\rangle=C_{\eta}^{\mu} \frac{\langle\eta|t| \eta+1\rangle\left\langle\eta+1 \mid \psi_{k}\right\rangle}{\epsilon_{k}-\epsilon_{\mu}} .
$$

At this point the second assumption is introduced. One writes

$$
\begin{aligned}
\left|\psi_{k}\right\rangle & \equiv(P+Q)\left|\psi_{k}\right\rangle \\
& =\sum_{\mu=1}^{\eta}|\mu\rangle\left\langle\mu \mid \psi_{k}\right\rangle+Q\left|\psi_{k}\right\rangle
\end{aligned}
$$

and assumes that within the range of the potential $Q\left|\psi_{k}\right\rangle$ is negligible relative to $P\left|\psi_{k}\right\rangle$. Such an as sumption is reasonable if $k$ is not so large that the number of nodes inside the potential is comparable to $\eta$. One then obtains, in this region,

$$
\left|\psi_{k}\right\rangle=\sum_{\mu}|\mu\rangle C_{\eta}^{\mu} \frac{\langle\eta|t| \eta+1\rangle}{\epsilon_{k}-\epsilon_{\mu}} a_{\eta+1}^{k},
$$

where

$$
a_{\eta+1}^{k}=\left\langle\eta+1 \mid \psi_{k}\right\rangle
$$

is a normalization constant. 
For Hermitian potentials, $\left|\psi_{k}\right\rangle$ should be orthogonal to the bound state wave functions. This implies that $C_{\eta}^{\mu}$ should be zero for these states. In practice, for reasonable values of $\eta$, the contribution to $\left|\psi_{k}\right\rangle$ from the bound states was negligible.

Although the wave function thus obtained is determined only within a multiplicative constant, one may still calculate the corresponding phase shift from it. Using the well-known expression

$$
\frac{-\tan \delta_{l}}{k}=\int_{0}^{\infty} j_{l}(k r) u(r) R_{k, l}(r) r^{2} d r,
$$

which is linear in the scattering solution, one may, using the Schrödinger equation, obtain the

expression 7
$\quad \frac{\tan \delta_{l}}{k}=\frac{\left[\int_{0}^{\infty} j_{l}(k r) u(r) R_{k, l}(r) r^{2} d r\right]^{2}}{\int_{0}^{\infty} R_{k, l}{ }^{2}(r) u(r) r^{2} d r-\int_{0}^{\infty} \int_{0}^{\infty} R_{k, l}\left(r^{\prime}\right) u\left(r^{\prime}\right) g_{k, l}\left(r^{\prime}, r\right) R_{k l}(r) u(r) r^{2} d r r^{\prime 2} d r^{\prime}}$,

which is independent of the normalization. Here $R_{k, l}$ is the radial part of $\left\langle r \mid \psi_{k}\right\rangle$ for a particular $l$ and

$$
g_{k, l}\left(r^{\prime}, r\right)= \begin{cases}k j_{l}\left(k r^{\prime}\right) n_{l}(k r), & r^{\prime}<r \\ k j_{l}(k r) n_{l}\left(k r^{\prime}\right), & r^{\prime}>r .\end{cases}
$$

This formula has a variational property and will yield phase shifts more accurate than the approximate wave functions $R_{k, l}$. Obviously one need only know the wave function within the potential region to utilize this method. The normalization constant can then be determined by using Eq. (16).

In summary, two assumptions are made:

$$
P u Q=0
$$

and

$$
\left|\left\langle r \mid Q \psi_{k}\right\rangle\right|^{2} \ll\left|\left\langle r \mid P \psi_{k}\right\rangle\right|^{2}
$$

within the range of the potential. As a result one may determine the scattering wave functions and the phase shifts extremely rapidly once the original matrix diagonalization has been affected.

The validity of the assumptions and the dependence on the size of the basis will be studied in the next section.

\section{APPLICATION: HARTREE-FOCK POTENTIAL}

As a first test of the method the Hartree-Fock potential for ${ }^{16} \mathrm{O}$, in a harmonic oscillator basis, was considered. The basis contained six $s$ states with $\gamma=\hbar / m \omega=3.7 \mathrm{fm}^{2}$. The Tabakin two-body interaction ${ }^{8}$ was used in the HF calculation. The exact solution was obtained by transforming the $6 \times 6$ potential into coordinate space and solving the Schrödinger equation with the resulting nonlocal potential using standard procedures.

The approximate scattering solutions were obtained as follows. The Hartree-Fock eigenfunctions and eigenvalues were used to obtain unnormalized scattering wave functions, Eq. (14), and the phase shifts and normalization were then determined via Eqs. (17) and (16). The results of these two calculations are compared in Table I. As is apparent from the table, the exact phase shifts are reproduced to within a fraction of a degree except at the lowest scattering energy. The results are for $s$-wave scattering and are typical of all partial waves considered. The corresponding wave functions are shown, for two energies, in Figs. 1 and 2. The accuracy of the method is well beyond what would be required in many-body calculations where the wave functions always enter into integrals restricted to the domain of the potential. The agreement strongly indicates that the assumptions involved in obtaining Eq. (14) are valid.

Because Hartree-Fock results in a larger basis were not available, the sensitivity to the size of the basis was tested as follows. A $10 \times 10$ " $\mathrm{Har}-$ tree-Fock" matrix was constructed using the $6 \times 6$ potential and the matrix elements of the kinetic energy. Diagonalization then produced 10 eigenfunctions and eigenvalues which were used to generate approximate scattering solutions. The phase shifts thus obtained are also listed in Table I. Obviously a $6 \times 6$ space is sufficiently large for this potential at the energies considered.

The method was also applied to a $3 \times 3$ HartreeFock potential ${ }^{1}$ for ${ }^{16} \mathrm{O}$ with similar agreement. In

TABLE I. Hartree-Fock potential. The phase shifts for $s$-wave scattering from the Hartree-Fock potential for ${ }^{16} \mathrm{O}$, as calculated in two different sized bases, are compared with the exact results.

\begin{tabular}{crrr}
\hline \hline$E(\mathrm{MeV})$ & $\delta$ (exact) & $\delta(6 \times 6)$ & $\delta(10 \times 10)$ \\
\hline 5 & 1.0240 & -0.6197 & -0.6200 \\
10 & -27.0609 & -27.1334 & -27.1333 \\
15 & -44.3904 & -44.9788 & -44.9788 \\
20 & -58.7131 & -58.7847 & -58.7846 \\
25 & -72.9702 & -72.8062 & -72.8064 \\
30 & -86.6877 & -86.7768 & -86.7768 \\
35 & 81.1252 & 80.8253 & 80.8253 \\
40 & 71.0551 & 70.7834 & 70.7834 \\
45 & 63.4058 & 63.2782 & 63.2783 \\
50 & 58.4475 & 58.4147 & 58.4147 \\
\hline \hline
\end{tabular}




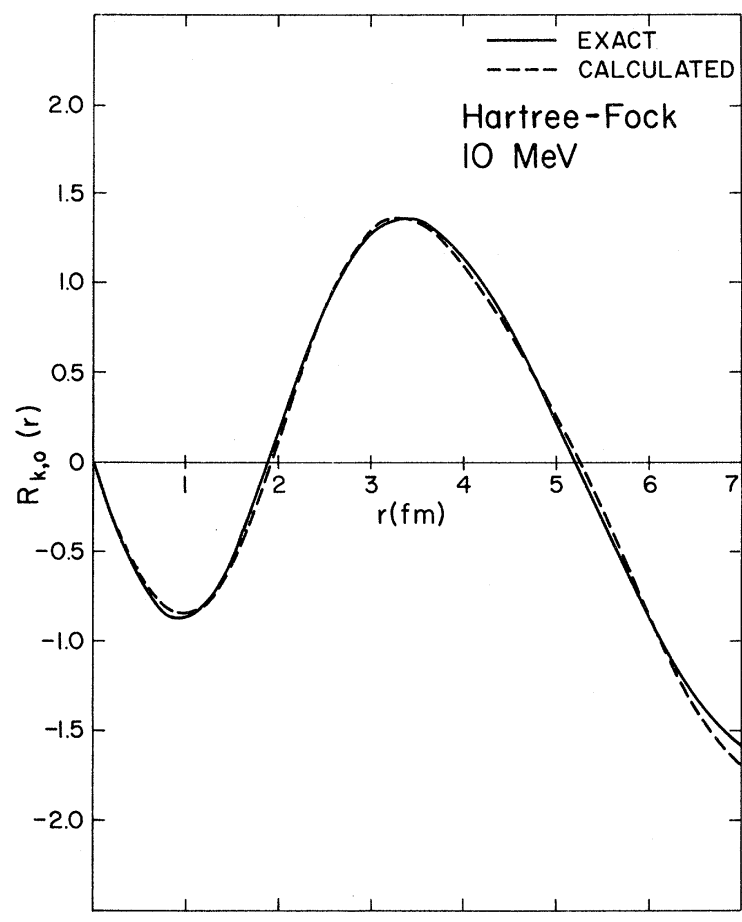

FIG. 1. Comparison of wave functions. The approximate wave function (dotted) is compared to the exact solution for $S$-wave scattering from the nonlocal Hartree-Fock potential at $10 \mathrm{MeV}$.

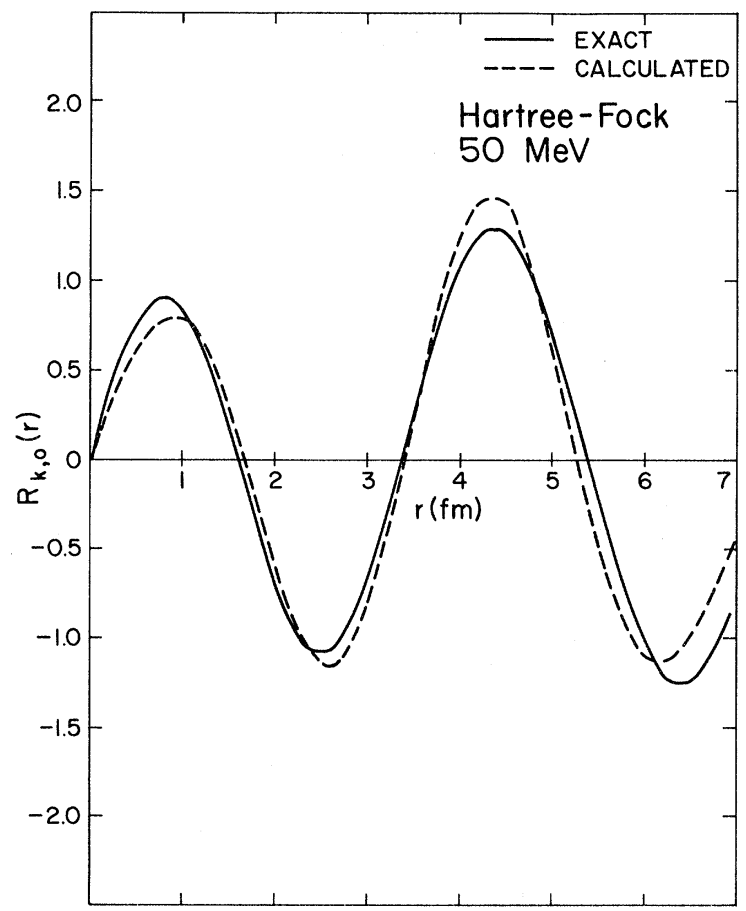

FIG. 2. Comparison of wave functions. The approximate wave function (dotted) is compared to the exact solution for $S$-wave scattering from the nonlocal Hartree-Fock potential at $50 \mathrm{MeV}$.

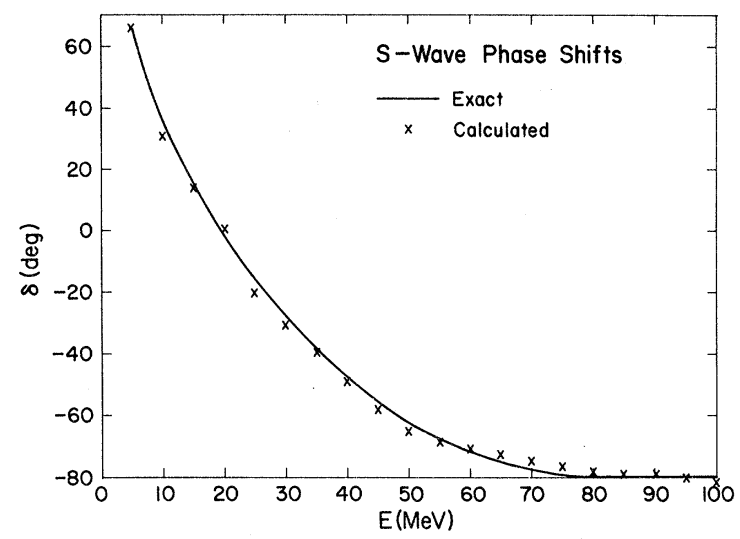

FIG. 3. S-wave scattering. The exact and approximate phase shifts are compared. Here a $10 \times 10$ space was utilized with $\gamma=2.6 \mathrm{fm}^{2}$. (The phase shifts are modulo $2 \pi$.)

that case the largest difference between the exact phase shifts and those calculated using a $10 \times 10$ space, from 5 to $50 \mathrm{MeV}$, was $0.1^{\circ}$.

\section{APPLICATION: SQUARE WELL}

As a final test of the method the three-dimensional square well was considered. This well was chosen both for simplicity and in an effort to make the test a severe one. Both the locality and the discontinuity in the well make it less amenable to this method than the $\mathrm{HF}$ potentials generated by smooth, two-body interactions.

The particular square well chosen had a depth of $60 \mathrm{MeV}$ and a radius of $2.9 \mathrm{fm}$. Such a well produces reasonable "single-particle energies" for the negative energy solutions if one is considering ${ }^{16} \mathrm{O} .{ }^{9}$ The scattering solutions and the expression

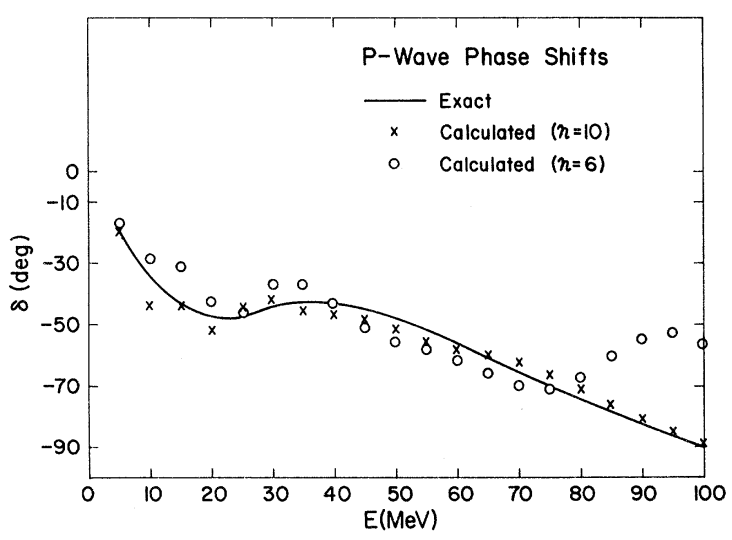

FIG. 4. Effect of truncation. The exact $P$-wave phase shifts are compared with the approximate solutions obtained in $10 \times 10$ and $6 \times 6$ spaces. The large deviations in the smaller space reflect the absence of eigenstates with large energies. 


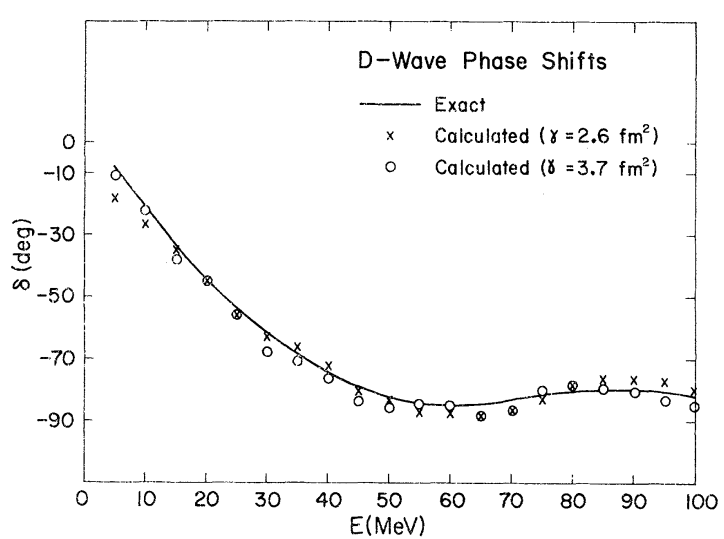

FIG. 5. Dependence on $\gamma$. The exact $D$-wave phase shifts are compared with the approximate solutions, in the $10 \times 10$ space, obtained using different harmonic oscillator constants.

for the phase shift are to be found in every quantum mechanics textbook. ${ }^{7}$ The approximate solutions were obtained by diagonalizing this Hamiltonian in an harmonic oscillator basis containing $\eta s$ states, $\eta p$ states, and $\eta d$ states, with $\eta$ taking on various values with a maximum of 10 . Although for this simple case much larger values of $\eta$ could have

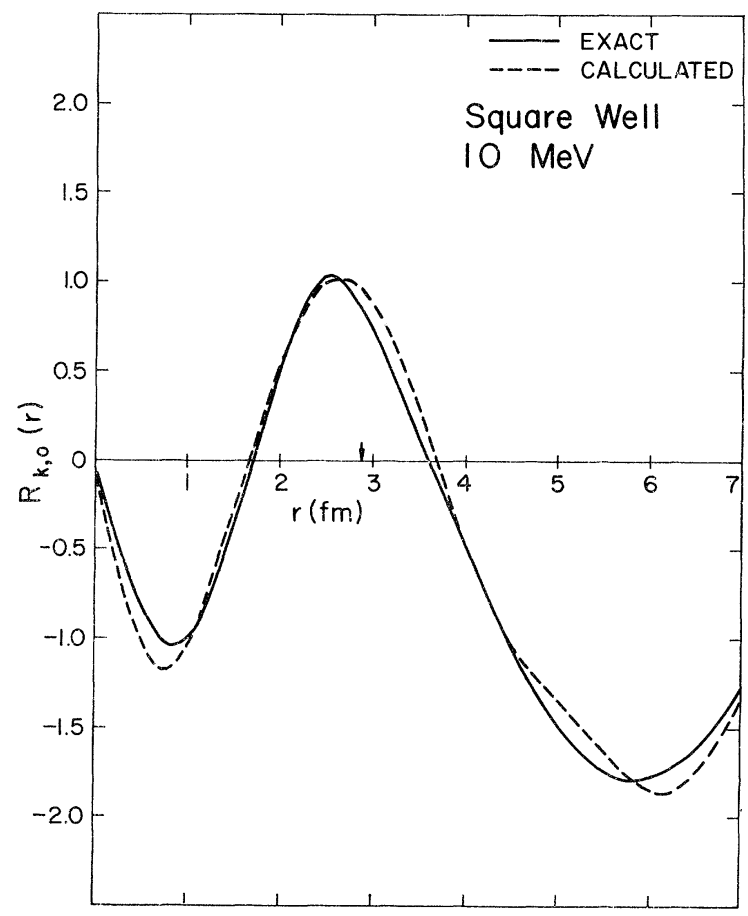

FIG. 6. Comparison of wave functions. The approximate wave function (dotted) is compared to the exact solution for $S$-wave scattering from a square well potential at $10 \mathrm{MeV}$. The arrow indicates the range of the potential. been easily used, such tests of the method would be irrelevant because of the limited bases used for bound state calculations.

The resulting phase shifts are compared to the exact results in Figs. 3-5. The first figure shows this comparison for $s$-wave scattering using a $10 \times 10$ space. Although the approximation works quite well, the errors here are quite a bit larger than for the Hartree-Fock potential. The reason for this, besides the pathological nature of the potential, is that one is now not using the same potential for the exact and approximate solutions. In contrast to the Hartree-Fock case, the potential used for the exact solution was not the coordinate space representation of the $10 \times 10$ potential, but the actual square well. Thus one is testing, as well as the method, the degree to which this potential can be represented in the truncated space. In fact, in the truncated space the potential will be nonlocal and this nonlocality was found to be significant relative to the local part. This renders the agreement shown in the figures more impressive.

In Fig. 4 the exact and approximate solutions are compared for $p$-wave scattering. Included in this figure are the results for a $6 \times 6$ space calculation. The agreement is again very good although the effects of the truncation become pronounced at higher

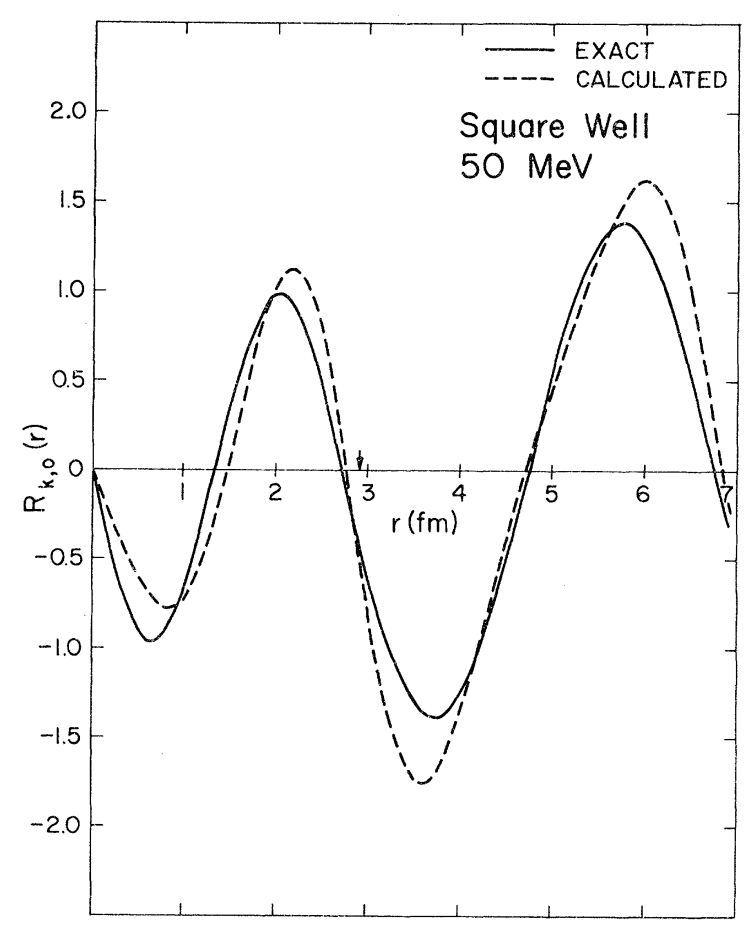

FIG. 7. Comparison of wave functions. The approximate wave function (dotted) is compared to the exact solution for $S$-wave scattering from a square well potential at $50 \mathrm{MeV}$. 
TABLE II. Square-well potential. The phase shifts calculated variationally, $\delta$ (calc), are compared with the exact phase shifts for a square well. The phase shifts labeled $\delta$ (fit) are obtained by matching the unnormalized approximate solution to the exact asymptotic form outside the range of the potential.

\begin{tabular}{rrrr}
\hline \hline$E(\mathrm{MeV})$ & $\delta$ (calc) & $\delta$ (exact) & $\delta$ (fit) \\
\hline 5 & 66.9 & 66.7 & 66.5 \\
10 & 30.7 & 36.5 & 38.0 \\
15 & 13.9 & 15.3 & 16.5 \\
20 & 0.0 & -1.6 & -1.1 \\
25 & -20.7 & -15.8 & -15.7 \\
30 & -31.3 & -28.0 & -28.1 \\
35 & -39.6 & -38.5 & -38.7 \\
40 & -48.8 & -47.7 & -47.9 \\
45 & -58.1 & -55.6 & -55.6 \\
50 & -65.1 & -62.3 & -61.6 \\
\hline \hline
\end{tabular}

energies. This is reasonable since it is the states with the larger eigenvalues which should dominate at higher energies and the energy range spanned by the $6 \times 6$ space is of course, smaller than for the $10 \times 10$ space. This observation provides a useful criterion for determining the size of the space based on the scattering energies desired.

In Fig. 5 the exact and approximate solutions are compared for $d$-wave scattering. Included are the approximate results for two quite different values of the harmonic oscillator parameter $\gamma$. In the Hartree-Fock case $\gamma$ was determined in the variational calculation so that the dependence on $\gamma$ was irrelevant. Here there is no unambiguous criterion for determining $\gamma$ so the weak dependence on it is gratifying.

The wave functions for $S$-wave scattering at two energies, with $\gamma=3.7 \mathrm{fm}^{2}$, are shown in Figs. 6 and 7. It should be noted that in most applications the wave functions would be multiplied by the potential which, in this case, is zero beyond $2.9 \mathrm{fm}$. It can be observed that there are no systematic discrepancies in these results due to the replacement of a local potential by a nonlocal one. ${ }^{10}$

\section{ALTERNATIVE METHOD}

Instead of carrying out the procedure described above one may, starting from the unnormalized approximate scattering states, utilize the known asymptotic form of the solution to determine scattering states. Assuming there is a region outside the potential for which Eq. (14) is valid, the normalization and the phase shift can be determined by requiring that they minimize the difference between the exact, asymptotic form [Eq. (8)] and the approximate solution. (Note that the last assumption is not required in applying the previous method.)

This two parameter search procedure ${ }^{11}$ is applicable to the square well considered above because there is a well-defined region, in which the potential is zero, which is close enough to the origin for Eq. (14) to be valid. The results, obtained by minimizing the difference between the solutions in a region of width $1.5 \mathrm{fm}$ starting from the edge of the well, are shown in Table II. As can be seen from the table, the $\delta$ (fit), obtained in this way, are somewhat more accurate than the $\delta$ (calc) obtained using Eq. (17). The computer time necessary for the two methods is comparable.

This alternative method is not relevant for the Hartree-Fock potential. There one must go beyond the range of validity of Eq. (14) before reaching a region in which the exact solution has taken on its asymptotic form. The alternative should, however, be considered when sharply cut-off, short-ranged potentials are being investigated.

\section{CONCLUSIONS}

A method has been proposed for expressing scattering solutions in terms of the eigenfunctions of a one-body Hamiltonian in a truncated harmonic oscillator basis. It has been demonstrated that even for a local, rather pathological potential the method reproduces the exact phase shifts and wave functions well. Furthermore, it has been shown that for the smooth, nonlocal Hartree-Fock potential, the method works extremely well. Because of the speed of the calculations the method is well suited for application in complex problems which require the one-body continuum for the construction of few-particle intermediate states. For example, the method provides a convenient way of representing the continuum states arising in the second order correction to the HF nucleon-nucleus optical potential, thus avoiding the plane-wave approximation.
${ }^{*}$ This work supported in part through funds provided by the National Science Foundation under grant No. GP38992 .

${ }^{1}$ A. K. Kerman, J. P. Svenne, and F. M. H. Villars, Phys. Rev. 147, 710 (1966).

${ }^{2}$ D. J. Thouless, Nucl. Phys. $\underline{22}, 78$ (1961).
${ }^{3}$ T. A. Brody and M. Moshinsky, Monografias del Institudo de Fisica, Mexico, 1960 (unpublished). ${ }^{4}$ A. M. Lane and R. G. Thomas, Rev. Mod. Phys. 30, 257 (1958).

${ }^{5} \mathrm{E}$. J. Heller and H. A. Yamani, in Electronic and Atomic Collisions, edited by B. C. Cobic and M. V. 
Kurepa (Institute of Physics, Beograd, Yugoslavia, 1973), Vol. 1, p. 5; E. J. Heller and H. A. Yamani, Phys. Rev. A 9, 1201 (1974).

${ }^{6}$ A. D. MacKellar, J. F. Reading, and A. K. Kerman, Phys. Rev. C $\underline{3}, 460$ (1971).

${ }^{7}$ See, for example, L. I. Schiff, Quantum Mechanics (McGraw-Hill, New York, 1955).

${ }^{8}$ F. Tabakin, Ann. Phys. (N.Y.) 30, 51 (1964).
${ }^{9}$ R. R. Scheerbaum, C. M. Shakin, and R. M. Thaler, Ann. Phys. (N.Y.) 76, 333 (1973).

${ }^{10}$ R. E. Schenter and A. D. MacKellar, Phys. Rev. C $\underline{4}$, 2020 (1971).

${ }^{11}$ The authors are grateful to Dr. Robert Graves of the Cyclotron Institute for supplying the curve-fitting computer program. 